CHRONIC OBSTRUCTIVE PULMONARY DISEASE

\title{
Adenine nucleotide loss in the skeletal muscles during exercise in chronic obstructive pulmonary disease
}

\author{
M C Steiner, R Evans, S J Deacon, S J Singh, P Patel, J Fox, P L Greenhaff, M D L Morgan
}

Thorax 2005;60:932-936. doi: 10.1136/thx.2004.038802

See end of article for authors' affiliations

Correspondence to:

Dr M C Steiner, Institute for Lung Health, Department of Respiratory Medicine, Glenfield Hospital, Groby Road, Leicester LE3 9QP, UK; michael.steiner@ uhl-tr.nhs.uk

Received

14 December 2004

Accepted 6 July 2005

Published Online First

29 July 2005
Background: Skeletal muscle adenine nucleotide loss has been associated with fatigue during high intensity exercise in healthy subjects but has not been studied in patients with chronic obstructive pulmonary disease (COPD). Changes in adenine nucleotides and other metabolites in the skeletal muscles were measured in patients with COPD and age matched healthy volunteers by obtaining biopsy samples from the quadriceps muscle at rest and following a standardised exercise challenge.

Methods: Eighteen patients with COPD (mean (SD) forced expiratory volume in 1 second 38.1 (16.8)\%) and eight age matched healthy controls were studied. Biopsy samples were taken from the vastus lateralis muscle at rest and immediately after a 5 minute constant workload cycle test performed at $80 \%$ peak work achieved during a maximal incremental cycle test performed previously.

Results: The absolute workload at which exercise was performed was substantially lower in the COPD group than in the controls (56.7 (15.9) W $v 143.2$ (26.3) W, $\mathrm{p}<0.01)$. Despite this, there was a significant loss of adenosine triphosphate (mean change $4.3(95 \% \mathrm{Cl}-7.0$ to -1.6$), \mathrm{p}<0.01$ ) and accumulation of inosine monophosphate $(2.03(95 \% \mathrm{Cl} 0.64$ to 3.42), $\mathrm{p}<0.01)$ during exercise in the COPD group that was similar to the control group $(-4.8(95 \% \mathrm{Cl}-9.7$ to 0.08$), p=0.053$ and $1.6(95 \%$ Cl 0.42 to 2.79 ), $p<0.01$, respectively).

Conclusions: These findings indicate that the ATP demands of exercise were not met by resynthesis from oxidative and non-oxidative sources. This suggests that significant metabolic stress occurs in the skeletal muscles of COPD patients during whole body exercise at low absolute workloads similar to those required for activities of daily living.
C hronic obstructive pulmonary disease (COPD) is an important and increasing cause of disability worldwide. ${ }^{1}$ It has been suggested that peripheral skeletal muscle dysfunction makes a significant contribution to whole body exercise intolerance in COPD. ${ }^{2}$ This is important because skeletal muscle dysfunction may be a remediable feature of an otherwise largely irreversible disease.

Recent studies of muscle samples taken from COPD patients at rest have shown reductions in oxidative enzyme activity and the proportion of type I fibres compared with healthy controls. ${ }^{3}$ This implies that resynthesis of ATP during muscle contraction from oxidative sources is impaired in COPD with a consequent increase in non-oxidative metabolism and presumably fatigue during exercise.

Severe metabolic stress can be said to occur in the skeletal muscles when the rate of ATP resynthesis from both oxidative and non-oxidative routes cannot meet its rate of utilisation during contraction. Under these circumstances, ATP loss and the accumulation of purine nucleotide derivatives such as inosine monophosphate (IMP) occur. This has been associated with fatigue during high intensity exercise in healthy subjects. ${ }^{56}$ However, it is unknown whether greater ATP loss and IMP accumulation occur in the muscles of COPD patients during whole body exercise, given the low absolute workloads patients are able to achieve.

Dynamic measurements of skeletal muscle energy metabolism in COPD can be made using magnetic resonance spectroscopy. ${ }^{7}$ However, magnetic resonance spectroscopy is unable to provide a complete metabolic profile of events during exercise and may be inaccurate because of assumptions made about the $\mathrm{pH}$ buffering capacity of the intracellular milieu, ${ }^{9}$ particularly in muscle where the fibre composition is likely to have changed. Moreover, whole body exercise cannot easily be studied using magnetic resonance spectroscopy because of the confines of the equipment

By contrast, the direct measurement of muscle metabolites from biopsy samples taken during whole body exercise is commonplace, particularly in studies of young healthy subjects. This allows a detailed examination of adenine nucleotide turnover and the accumulation of purine nucleotide metabolites. In addition, the contribution of oxidative and non-oxidative pathways to the resynthesis of ATP during muscular contraction can be accurately quantified. This has not been performed in patients with COPD and therefore the functional relevance of adenine nucleotide turnover during exercise to fatigue and exercise limitation is unclear.

In this study we have examined the metabolic response to exercise by analysing biopsy samples taken at rest and during a standardised exercise challenge in COPD patients and similar aged healthy volunteers. Our aim was to determine whether significant adenine nucleotide loss occurs in the muscles of COPD patients during exercise that is of functional relevance to daily activity and rehabilitation.

\section{METHODS \\ Patients}

Subjects were recruited from those accepted for pulmonary rehabilitation at Glenfield Hospital. Patients who met GOLD

Abbreviations: ADP, adenosine diphosphate; AMP, adenosine monophosphate; ATP, adenosine triphosphate; COPD, chronic obstructive pulmonary disease; $F E V_{1}$, forced expiratory volume in 1 second; FVC, forced vital capacity; IMP, inosine monophosphate; MVV, maximum voluntary ventilation; PCr, phosphocreatine; SLP, substrate level phosphorylation; TAN, total adenine nucleotide 
clinical and spirometric criteria for COPD between the ages of 40 and 80 years of age were included. ${ }^{10}$ All patients had agreed to participate in a nutritional supplementation trial during pulmonary rehabilitation ${ }^{11}$ and were therefore excluded if their body mass index was greater than $30 \mathrm{~kg} /$ $\mathrm{m}^{2}$. The current study was completed before rehabilitation and supplementation commenced. Healthy age matched volunteers were recruited as controls by local advertisement. Healthy controls had no past or present history of cardiac or respiratory disease and had normal spirometic parameters. Other exclusion criteria were oral corticosteroid or anticoagulant use and resting or exercise induced haemoglobin desaturation below $90 \%$.

Approval for the study was obtained from the Leicestershire research ethics committee.

\section{Study assessments}

Spirometric parameters were measured in the seated position to UK standards (Vitalograph, Model R, Bucks, UK). Values are expressed as a percentage of predicted values calculated from ERS regression equations. ${ }^{12}$ Body weight was measured in light clothing to the nearest $0.1 \mathrm{~kg}$ (SECA, UK). Body height was measured using a wall mounted stadiometer to the nearest $1 \mathrm{~cm}$.

\section{Exercise tests}

All subjects performed a maximal symptom limited incremental exercise test on an electrically braked cycle ergometer to determine peak exercise work capacity. Patients were asked to cycle at a constant speed of $40 \mathrm{rpm}$. Following a 3 minute period of unloaded cycling, the workload was increased incrementally by $10 \mathrm{~W}$ every minute. Patients were encouraged to continue cycling at the required rate for as long as possible. The peak work achieved was recorded. During the test ventilation and gas exchange measurements were made using a breath by breath computerised system (Oxycon Beta, Erich, Jaeger UK Ltd). Peak ventilation was also expressed as a percentage of predicted maximum voluntary ventilation (MVV). This was calculated as forced expiratory volume in 1 second $\left(\mathrm{FEV}_{1}\right) \times 40$, and patients with COPD were deemed to have a ventilatory limit to maximal exertion if peak ventilation was greater than $90 \%$ MVV. ${ }^{13}$

At least 48 hours after the incremental cycle test subjects performed a 5 minute constant load cycle test. The workload for this test was set at $80 \%$ of the peak work achieved during the incremental test. The cycle cadence for all exercise tests was $40 \mathrm{revs} / \mathrm{min}$. Muscle biopsy samples were obtained from the quadriceps muscle at rest and at the end of the constant load test using the Bergstrom technique. ${ }^{14}$ Muscle samples were therefore taken from all subjects following an exercise challenge of the same time and relative intensity. Post exercise samples were obtained with the subject seated in the bike, no longer than 10 seconds after the end of the exercise test. This is effectively an exercising sample. ${ }^{14}$ ECG and saturation monitoring was performed throughout the test. Breathlessness and perceived exertion were assessed on the modified Borg scale at the end of the test. Breath by breath measurements of ventilation and gas exchange were not obtained during the constant load test. The reason for this was to facilitate rapid sampling of the muscle within a few seconds of the completion of exercise. This technique is used routinely in studies of young healthy subjects and we were able to obtain samples for all subjects without difficulty within 10 seconds after exercise.

\section{Muscle biopsy analysis}

Muscle samples were frozen immediately and stored in liquid nitrogen. Following subsequent freeze drying, powdering and extraction, samples were analysed for phosphocreatine (PCr), creatine, and lactate concentrations using the spectrophotometric method of Harris et al, ${ }^{15}$ and adenine nucleotides (ATP, ADP and AMP) and their breakdown derivatives (IMP, inosine and xanthine) using high pressure liquid chromatography (HPLC). ${ }^{16}$ Total creatine concentration was calculated as the sum of PCr and creatine and all measurements (apart from lactate which in part leaves the muscle compartment) were corrected for non-muscle constituents using total creatine. ${ }^{17}$

Substrate level phosphorylation (SLP) was calculated as $\mathrm{SLP}=\Delta[\mathrm{PCr}]+\{\Delta[\mathrm{La}] \times 1.5\}+\{\Delta[\mathrm{ATP}] \times 2\}$ where $\Delta$ refers to the exercise induced change in each metabolite. Substrate level phosphorylation represents total ATP energy derived from non-oxidative sources. As oxidative metabolism is the only other source of ATP energy, its contribution to energy metabolism can be indirectly assessed by calculating SLP. ${ }^{18}$ The total adenine nucleotide (TAN) pool was calculated as $[\mathrm{ATP}]+[\mathrm{ADP}]+[\mathrm{AMP}]$.

To allow comparison of the metabolic response to exercise between patients and controls working at markedly different absolute intensities, SLP and IMP accumulation during exercise was adjusted by dividing by the workload at which the constant load test was performed (SLP/WL and IMP/WL, respectively).

\section{Data analysis}

Comparison between patients and controls was made using the Student's unpaired $t$ test. Association between variables was analysed by Pearson correlation. Statistical tests were performed using SPSS Version 10 (SPSS Inc, Chicago, USA).

\section{RESULTS}

Twenty three patients and 10 healthy controls took part in the study. Complete paired muscle biopsy samples (rest and exercise) were available from 18 patients with COPD and eight healthy controls. Missing data were due to insufficient muscle tissue for accurate analysis in either the rest or exercise biopsies. Only data from patients with rest and exercise samples were included in the analysis. The baseline subject characteristics are shown in table 1.

Apart from spirometric measurements, the patient and control groups were demographically well matched at baseline. Data from the maximal and constant load tests are shown in table 2. Exercise data from three patients in the COPD group are missing because of equipment failure. As expected, peak workload, peak oxygen uptake, and peak ventilation during incremental exercise were substantially higher in the control group. Peak ventilation as a proportion of predicted MVV was significantly higher in the COPD group. Of the 15 patients in the COPD group for whom data were available, nine were deemed ventilatory limited by our criteria. During the constant load test the end exercise Borg breathlessness score was higher in the COPD group despite

\begin{tabular}{|c|c|c|}
\hline & $\begin{array}{l}\text { COPD patients } \\
(n=18)\end{array}$ & $\begin{array}{l}\text { Healthy controls } \\
(\mathrm{n}=8)\end{array}$ \\
\hline$F / M$ & $3 / 15$ & $2 / 6$ \\
\hline Age (years) & $66(8.1)$ & $64(6.1)$ \\
\hline $\mathrm{FEV}_{1}$ (I) & $1.12(0.4)$ & $2.92(0.62)^{*}$ \\
\hline $\mathrm{FEV}_{1}$ (\% predicted) & $38.1(16.8)$ & $100.9(21.6)^{*}$ \\
\hline FVC (l) & $3.02(0.73)$ & $3.57(0.81)$ \\
\hline $\mathrm{SpO}_{2}$ at rest (\%) & 95.4 (1.5) & $97.6(1.06)^{*}$ \\
\hline BMI $\left(\mathrm{kg} / \mathrm{m}^{2}\right)$ & 24.7 (4.2) & $25.2(2.6)$ \\
\hline
\end{tabular}

$\mathrm{FEV}_{1}$, forced expiratory volume in 1 second; FVC, forced vital capacity; $\mathrm{SpO}_{2}$, oxygen saturation; $\mathrm{BMI}$, body mass index. ${ }^{*} p<0.001$ compared with COPD patients. 
Table 2 Exercise data from incremental and constant load cycle tests

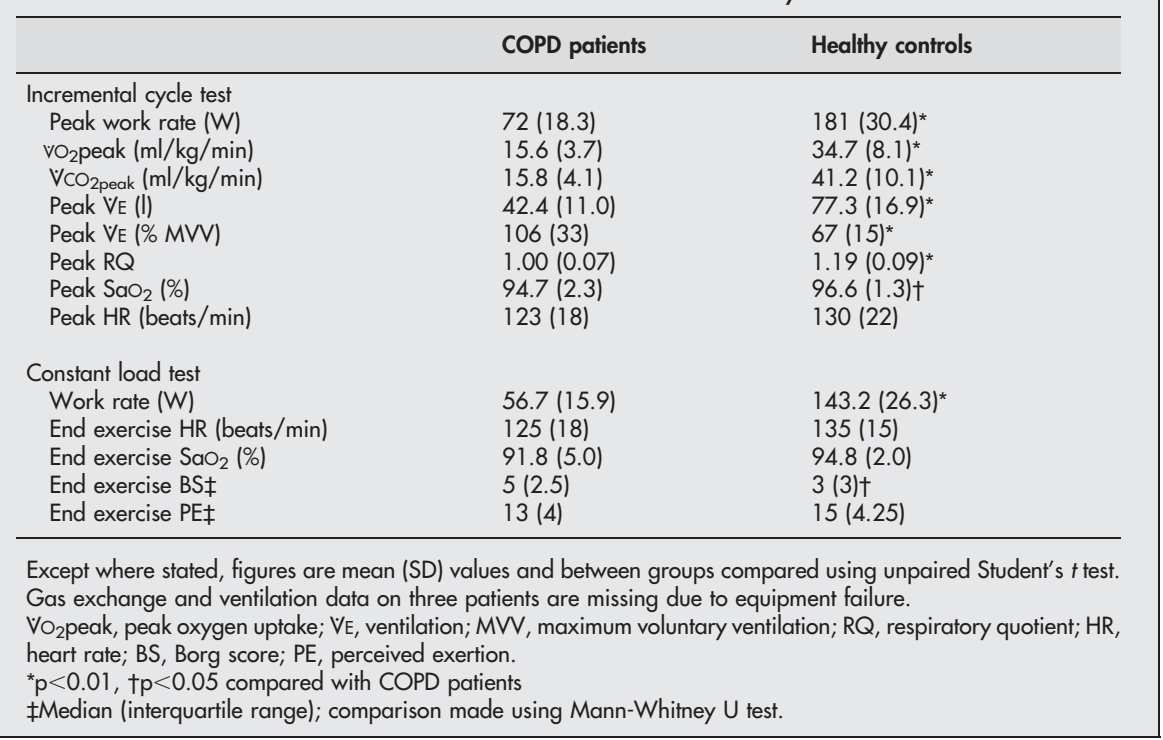

the significantly lower workloads at which exercise was performed.

Metabolite levels measured in muscle samples taken at rest and exercise and the exercise induced change in muscle metabolites in the COPD and control groups are shown in table 3 and fig 1 . There were significant differences between COPD patients and controls in resting concentrations of lactate, ATP, total adenine nucleotide (TAN) pool, and purine nucleotide derivatives (table 3 ). In both groups there was significant PCr degradation and lactate accumulation during exercise. In addition, both groups showed a decline in ATP concentration during exercise which was statistically
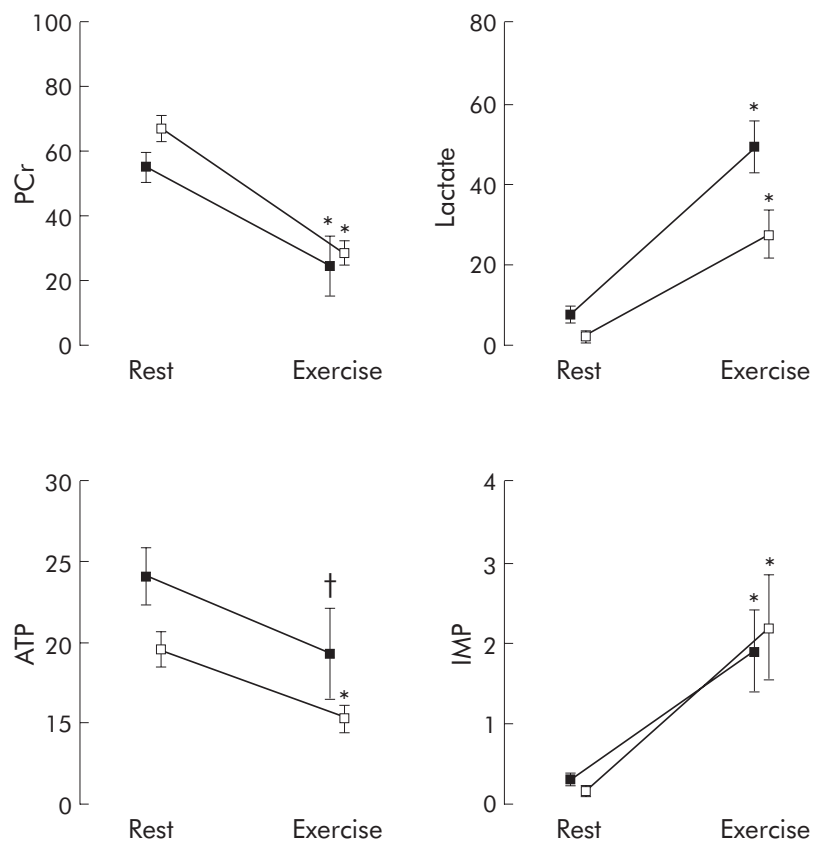

Figure 1 Exercise induced changes in muscle metabolites. Mean (SE) muscle metabolite values taken at rest and after exercise in COPD patients (open symbols) and healthy controls (solid symbols). See table 3 for figures. All units $\mathrm{mmol} / \mathrm{kg}$ dry weight. ${ }^{*} \mathrm{p}<0.001 ; \mathrm{tp}=0.053$ (comparisons made with resting values using paired Student's $t$ test). $\mathrm{PCr}$, phosphocreatine; ATP, adenosine triphosphate; IMP, inosine monophosphate. significant for COPD patients with a strong trend in the control group $(p=0.053)$. Similarly, there was a statistically significant decline in the TAN pool following exercise in the COPD group with a strong trend in the control group $(p=0.063)$. As might be expected from the changes in muscle ATP concentration, exercise also resulted in a significant accumulation of IMP in both groups (table 3, fig 1). There were no statistically significant differences between the groups in magnitude of the exercise induced changes in muscle metabolites despite the marked differences in workload. Mean (SD) substrate level phosphorylation was similar in both groups (87 (57) mmol ATP/kg dry weight in patients with COPD $v 107$ (44) mmol ATP/kg dry weight in controls, $\mathrm{p}=0.351)$, but work adjusted SLP was significantly higher in COPD patients ( 1.58 (1.07) mmol ATP/ $\mathrm{kg} / \mathrm{W})$ than in controls $(0.78(0.39) \mathrm{mmol} \mathrm{ATP} / \mathrm{kg} / \mathrm{W} ; \mathrm{p}<0.01$, fig 2). There was a trend towards a greater rise in IMP during exercise when adjusted for workload (mean (SD) values for COPD group: $0.042(0.01) \mathrm{mmol} / \mathrm{kg} / \mathrm{W} v$ controls: 0.012 (0.004) $\mathrm{mmol} / \mathrm{kg} / \mathrm{W} ; \mathrm{p}=0.075$, fig 2 ).

$\mathrm{PCr}$ utilisation correlated with lactate accumulation $(r=0.597, \mathrm{p}=0.001)$ but not with loss of ATP $(r=-0.294$, $\mathrm{p}=0.124)$. The accumulation of IMP correlated with the decline in $\mathrm{PCr}(r=-0.562, \mathrm{p}=0.003)$. Exercise induced changes in muscle metabolites did not correlate with exercise workload, peak oxygen uptake, or indices of airflow obstruction. The metabolic response to exercise did not differ between ventilatory limited and non-ventilatory limited COPD patients.

\section{DISCUSSION}

This study is the first to evaluate the skeletal muscle response to whole body exercise in patients with COPD by directly measuring changes in intramuscular metabolites. The sampling of the vastus lateralis muscle during exercise allows the accurate quantification of ATP turnover, activation of the purine nucleotide cycle, and the contribution of oxidative and non-oxidative pathways to ATP production. This is not provided by measurements taken at rest or by magnetic resonance spectroscopy.

This study shows that significant adenine nucleotide loss occurs in the muscles of COPD patients during a short constant load exercise test at $80 \%$ peak capacity. In keeping with this, there was significant activation of SLP and accumulation of IMP during exercise. The activation of SLP 

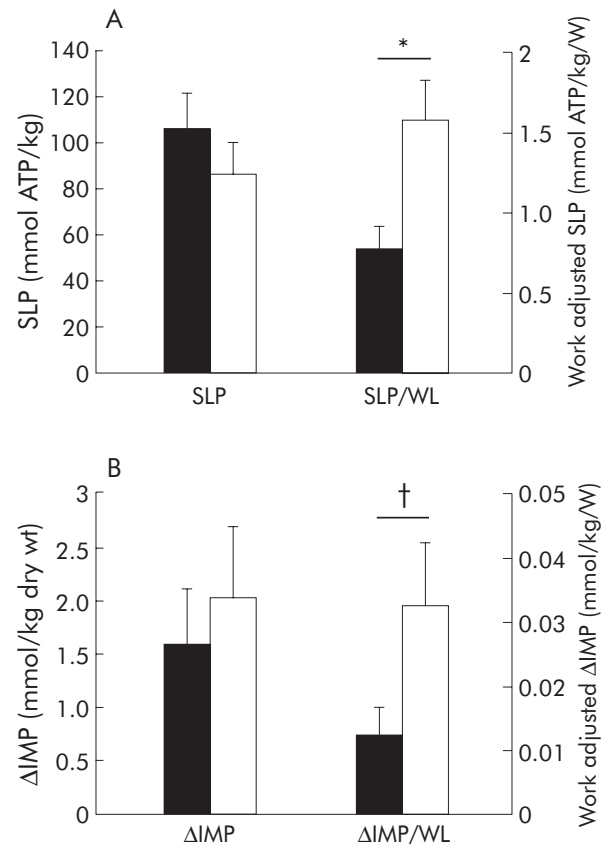

Figure 2 Substrate level phosphorylation (SLP) and inosine monophosphate (IMP) accumulation during exercise in COPD patients and controls. (A) Mean (SE) values for absolute SLP and work adjusted substrate level phosphorylation (SLP/WL) in COPD patients (open bars) and healthy controls (solid bars). (B) Exercise induced change in absolute ( $\triangle \mathrm{IMP}$ ) and work adjusted IMP ( $\triangle \mathrm{IMP} / \mathrm{WL}$ ) in COPD patients (open bars) and healthy controls (solid bars). ${ }^{*} \mathrm{p}<0.01 ; \mathrm{tp}=0.075$.

in these circumstances indicates the mobilisation of nonoxidative energy production due to the failure of oxidative metabolism to meet the demand for ATP. The accumulation of IMP is due to the irreversible deamination of AMP and occurs when ATP resynthesis is unable to meet demand. In the short term this increases the phosphorylation potential of the adenine nucleotide pool, allowing the adenylate kinase reaction to continue. Such a situation is not sustainable, however, because of the resulting decline in the total adenine nucleotide pool and ATP availability. Collectively, these data indicate that oxidative and non-oxidative resynthesis of ATP was unable to meet ATP demand during the exercise challenge used in this study. Substantial metabolic "stress" can be said to have occurred under these conditions because the imbalance between ATP utilisation and resynthesis results in a progressive decline in the availability of ATP required to sustain contraction. Adenine nucleotide loss and the irreversible formation of IMP has been associated with fatigue in healthy subjects during high intensity or prolonged exercise. ${ }^{56}$

A striking observation of this study was the similarity of the metabolic response to exercise between the COPD group and controls. This occurred despite the COPD group working at substantially lower absolute exercise workloads. While the activation of SLP and accumulation of purine nucleotide derivatives (principally IMP) was similar in absolute terms, when these figures were adjusted for the workload at which exercise was performed there were marked numerical differences between the COPD and healthy control groups. This was statistically significant for work adjusted SLP, with a strong trend being observed for work adjusted IMP accumulation. In other words, in the COPD group, a given amount of work resulted in greater SLP activation and adenine nucleotide loss than in the healthy control group.

Our findings are compatible with previous data from skeletal muscle samples taken at rest that have indicated reductions in oxidative enzyme concentrations and alterations in fibre composition in COPD patients. ${ }^{3}{ }^{4}$ This implies a reduction in oxidative and an increase in non-oxidative sources of energy during exercise in COPD. Further support for this notion has been provided by studies showing accelerated lactate release in femoral venous blood during exercise $^{19}$ and magnetic resonance spectroscopic studies, which demonstrated slower PCr resynthesis following exercise. $^{7}$ Importantly, our data show that this occurs during whole body exercise even at the low absolute work intensities achieved by COPD patients. We have also shown that, despite the substantial mobilisation of non-oxidative sources of energy, ATP resynthesis was unable to meet demands of exercise resulting in adenine nucleotide loss and the accumulation of IMP.

Our data are consistent with previous measurements of metabolites in muscle samples taken at rest, which showed lower levels of ATP in patients with COPD. ${ }^{20-22}$ However, our finding of lower resting levels of lactate and purine nucleotide derivatives in the COPD group contrasts with these previous studies. We cannot determine the reasons for these differences, although many of the patients studied in these earlier reports had respiratory failure whereas this was an exclusion criterion for the current study. Technical differences in the sampling and analysis of muscle tissue may also have contributed to these differences. It is also

Table 3 Muscle metabolites at rest and exercise

\begin{tabular}{|c|c|c|c|c|}
\hline & \multicolumn{2}{|c|}{ COPD patients ( $n=18$ ) } & \multicolumn{2}{|c|}{ Healthy controls $(n=8)$} \\
\hline & Rest & $\begin{array}{l}\text { Exercise induced } \\
\text { change }(95 \% \mathrm{Cl})\end{array}$ & Rest & $\begin{array}{l}\text { Exercise induced } \\
\text { change }(95 \% \mathrm{Cl})\end{array}$ \\
\hline $\mathrm{PCr}$ & $67.9(17.1)$ & $-38.1(-48.3 \text { to }-27.9)^{*}$ & $55.4(12.7)$ & $-30.6(-45.5 \text { to }-15.8)^{*}$ \\
\hline Lactate & $2.3(1.5)$ & $25.7(12.4 \text { to } 39.0)^{*}$ & $7.5(4.7)^{*}$ & $42.1(26.8 \text { to } 57.4)^{*}$ \\
\hline ATP & $19.5(4.5)$ & $-4.3(-7.0 \text { to }-1.6)^{*}$ & $24.1(4.9) \dagger$ & $-4.8(-9.7$ to 0.08$) \ddagger$ \\
\hline ADP & $2.67(0.87)$ & $0.53(-0.03$ to 1.09$)$ & $3.1(1.2)$ & $-0.03(-0.84$ to 0.79$)$ \\
\hline AMP & $0.03(0.03)$ & $0.03(-0.02$ to 0.08$)$ & $0.24(0.17)^{*}$ & $-0.02(-0.21$ to 0.18$)$ \\
\hline TAN pool & $22.2(5.2)$ & $-3.7(-6.8$ to -0.7$) \dagger$ & $27.4(6.0) \dagger$ & $-4.8(-10$ to 0.3$) \S$ \\
\hline IMP & $0.14(0.06)$ & $2.03(0.64 \text { to } 3.42)^{*}$ & $0.28(0.09)^{*}$ & $1.6(0.42 \text { to } 2.79)^{*}$ \\
\hline Inosine & $0.73(1.05)$ & $-0.26(-0.75$ to 0.24$)$ & $2.06(0.30)^{*}$ & $-0.17(-0.58$ to 0.24$)$ \\
\hline Xanthine & $0.07(0.03)$ & $-0.01(-0.03$ to 0.01$)$ & $0.38(0.08)^{*}$ & $-0.02(-0.15$ to 0.10$)$ \\
\hline
\end{tabular}

PCr, phosphocreatine; ATP, adenosine triphosphate; ADP, adenosine diphosphate; AMP, adenosine monophosphate; IMP, inosine monophosphate; TAN pool, total adenine nucleotide pool.

Resting figures are mean (SD) values. Mean (95\% confidence interval) changes are shown. Negative values denote a decrease after exercise.

All units are $\mathrm{mmol} / \mathrm{kg}$ dry wt. Symbols of statistical significance for resting values refer to comparisons between COPD and control groups using the unpaired Student's $t$ test. Symbols of statistical significance for the response to exercise refer to within subject comparison of metabolite changes using the paired Student's $t$ test. There were no statistically significant differences in the exercise induced changes in metabolites between the COPD and control groups. ${ }^{*} \mathrm{p}<0.01 ; \mathrm{tp}<0.05 ; \mathrm{f}=0.053 ; \S p=0.063$. 
noteworthy that, although differences in resting lactate and IMP between the COPD and control groups were statistically significant, the magnitude of these differences was small when compared with the exercise induced change. More research will be needed for these questions to be settled.

A number of limitations to the current study should be noted. We observed considerable inter-individual variability in the COPD group in the degree of metabolic response observed during exercise. This was less evident in the healthy control group. Because the workload for the constant load test was determined by performance during the maximal incremental test, the metabolic response to exercise measured in the muscles will have been influenced by the limit to maximal performance. While the majority of our patients had ventilatory limitation, other factors such as muscle fatigue and individual effort may also have been important. This is supported by our observation during the conduct of the study that some patients found completing 5 minutes of cycling at $80 \%$ peak capacity extremely taxing while others appeared to do so with relative ease. This variability in response highlights the difficulty in standardising submaximal exercise tests for studies in COPD. Similarly, direct comparison with healthy controls is limited because of large differences in absolute exercise intensity. For practical reasons we did not determine the magnitude of the metabolic response in healthy subjects at similar absolute workloads to the COPD group. We were unable to predict the magnitude of the metabolic response from baseline demographic, lung function, or exercise test variables. We recognise, however, that this was not an exhaustive assessment and that the sample size may have been too small to detect such relationships. Finally, we can only speculate about the mechanisms underlying our findings. An increase in the proportion of type II fibres has been reported in patients with $\mathrm{COPD}^{4}$ and this may have been particularly relevant as these fibres are more susceptible to ATP loss and IMP accumulation during exercise. $^{5}$ Similarly, central factors such as muscle blood flow and oxygen delivery and intramuscular changes in oxidative enzyme activity, mitochondrial density, and capillarity may have been important.

Our data show that, for many patients with COPD, significant metabolic stress occurs in the skeletal muscles despite the low exercise workloads that these patients can achieve and despite the ventilatory constraints to exercise evident in most of our subjects. This supports the notion that, even for those patients with severe lung function impairment, the metabolic load to ventilation from the exercising muscles is an important factor limiting exercise performance (at least during cycling) in this population. For healthy subjects, exercising at $80 \%$ peak capacity would be relatively unusual, but this is unlikely to be the case for patients who are regularly required to work near the ceiling of maximal exercise capacity to perform routine daily activities. Moreover, the findings are particularly relevant for pulmonary rehabilitation where exercise is performed frequently at high relative workloads. Indeed, the exercise protocol in the current study was chosen in part because it reflects exercise prescription during pulmonary rehabilitation at our institution. We are not able from our data to infer that metabolic stress is the limit to constant load exercise in our patients because biopsies were taken at a specified time point rather than at the limit of performance. More studies will be needed to determine the wider significance of our findings to exercise limitation and disability in COPD.

In conclusion, we have shown that significant metabolic stress occurs in the muscles of patients with COPD during whole body exercise. The metabolic response to exercise was of similar magnitude to healthy controls, despite the low workloads that patients achieved. Our results suggest that skeletal muscle energy metabolism may be an important factor in exercise limitation and disability in COPD.

\section{Authors' affiliations}

M C Steiner, R Evans, S J Deacon, S J Singh, P Patel, M D L Morgan, Institute for Lung Health, Department of Respiratory Medicine, Glenfield Hospital, Leicester, UK

J Fox, P L Greenhaff, Centre for Integrated Systems Biology and Medicine, Department of Biomedical Sciences, University of Nottingham, UK

Partial financial support for this independent study was provided by Nutricia, Zoetermeer, The Netherlands.

Competing interests: none declared

\section{REFERENCES}

1 Murray CJ, Lopez AD. Alternative projections of mortality and disability by cause 1990-2020: Global Burden of Disease Study. Lancet 1997;349:1498-504.

2 American Thoracic Society. Skeletal muscle dysfunction in chronic obstructive pulmonary disease. A statement of the American Thoracic Society and European Respiratory Society. Am J Respir Crit Care Med 1999;159:S1-40.

3 Maltais $F$, LeBlanc $P$, Whittom $F$, et al. Oxidative enzyme activities of the vastus lateralis muscle and the functional status in patients with COPD. Thorax 2000:55:848-53.

4 Whittom F, Jobin J, Simard PM, et al. Histochemical and morphological characteristics of the vastus lateralis muscle in patients with chronic obstructive pulmonary disease. Med Sci Sports Exerc 1998;30:1467-74.

5 Sahlin K, Broberg S, Ren JM. Formation of inosine monophosphate (IMP) in human skeletal muscle during incremental dynamic exercise. Acta Physiol Scand 1989;136:193-8

6 Broberg S, Sahlin K. Adenine nucleotide degradation in human skeleta muscle during prolonged exercise. J Appl Physiol 1989;67:116-22.

7 Sala E, Roca J, Marrades RM, et al. Effects of endurance training on skeletal muscle bioenergetics in chronic obstructive pulmonary disease. Am J Respir Crit Care Med 1999; 159:1726-34.

8 Kutsuzawa T, Shioya S, Kurita D, et al. Muscle energy metabolism and nutritional status in patients with chronic obstructive pulmonary disease. $A^{31} \mathrm{P}$ magnetic resonance study. Am J Respir Crit Care Med 1995;152:647-52.

9 Constantin-Teodosiu D, Greenhaff PL, Mclntyre DB, et al. Anaerobic energy production in human skeletal muscle in intense contraction: a comparison of ${ }^{31} \mathrm{P}$ magnetic resonance spectroscopy and biochemical techniques. Exp Physiol 1997; 82:593-601.

10 Global Initiative for Chronic Obstructive Lung Disease. Global strategy for the diagnosis, management and prevention of COPD, NIH Publication No 2701.NHLBI/WHO Workshop Report. Bethesda, MD: National Heart, Lung and Blood Institute, 2001.

11 Steiner MC, Barton RL, Singh SJ, et al. The nutritional enhancement of exercise performance in chronic obstructive pulmonary disease. A randomised controlled trial. Thorax 2003;58:745-51.

12 Quanjer PH, Tammeling GJ, Cotes JE, et al. Lung volumes and forced ventilatory flows. Report Working Party Standardization of Lung Function Tests, European Community for Steel and Coal. Official Statement of the European Respiratory Society. Eur Respir J Suppl 1993;16:5-40.

13 Cooper CB, Storer TW. Exercise testing and interpretation. A practical approach. Cambridge: Cambridge University Press, 2001

14 Bergstrom J. Percutaneous needle biopsy of skeletal muscle in physiological and clinical research. Scand J Clin Lab Invest 1975;35:609-16.

15 Harris RC, Hultman E, Nordesjo LO. Glycogen, glycolytic intermediates and high-energy phosphates determined in biopsy samples of musculus quadriceps femoris of man at rest. Methods and variance of values. Scand J Clin Lab Invest 1974;33:109-20.

16 Wynants J, Van Belle H. Single-run high-performance liquid chromatography of nucleotides, nucleosides, and major purine bases and its application to different tissue extracts. Anal Biochem 1985;144:258-66.

17 Hultman E, Sjoholm H. Energy metabolism and contraction force of human skeletal muscle in situ during electrical stimulation. J Physiol 1983;345:525-32.

18 Spriet LL, Soderlund K, Bergstrom M, et al. Anaerobic energy release in skeletal muscle during electrical stimulation in men. J Appl Physiol 1987;62:611-5.

19 Maltais F, Jobin J, Sullivan MJ, et al. Metabolic and hemodynamic responses of lower limb during exercise in patients with COPD. J Appl Physiol 1998;84:1573-80.

20 Pouw EM, Schols AM, van der Vusse GJ, et al. Elevated inosine monophosphate levels in resting muscle of patients with stable chronic obstructive pulmonary disease. Am J Respir Crit Care Med 1998;157:453-7.

21 Jakobsson P, Jorfeldt L, Brundin A. Skeletal muscle metabolites and fibre types in patients with advanced chronic obstructive pulmonary disease (COPD), with and without chronic respiratory failure. Eur Respir J 1990;3:192-6.

22 Moller P, Bergstrom J, Furst $P$, et al. Energy-rich phosphagens, electrolytes and free amino acids in leg skeletal muscle of patients with chronic obstructive lung disease. Acta Med Scand 1982;211:187-93. 